\title{
Representations of the CAR Generated by Representations of the CCR. Fock Case
}

\author{
Piotr Garbaczewski \\ Institute of Theoretical Physics, University of Wrocław, Wrocław, Poland
}

Received August 27, 1974; in revised form March 11, 1975

\begin{abstract}
We present a method of constructing the Fock representation of the canonical anticommutation relations in the Fock representation of the canonical commutation relations. An explicit formula for Fermi creation and annihilation operators in terms of Bose ones is given.
\end{abstract}

\section{Introduction}

The spinor theory of Heisenberg [1] is an example of the philosophy that a fundamental theory of elementary particles must involve Fermi rather than Bose fields in the basic formalism. Quite the contrary, there were many attempts $[2,3]$ and references there-in to describe fermions in terms of bosons. Streater and Wilde [2] have shown that in two-dimensional space-time fermion states of a boson field do exist.

Kalnay, MacCotrina and Kademova [3] succeeded to show that in the case of Fock representations, free Fermi field can be expanded into a sum of pairs of Bose operators.

We want to present an independent investigation showing that certain homomorphisms of the $n$-th power space $K^{\otimes n}$ can be utilized to produce the Fock representations of the CAR algebra expressed by infinite series in Bose creation and annihilation operators.

\section{Notations}

Let $\mathscr{K}, \mathscr{H}$ be complex Hilbert spaces with an involution $*, \mathscr{K} \ni f, g$. Let $\mathscr{U}_{F}(\mathscr{K})$ be a $*$ representation of the CAR algebra over $\mathscr{K}$ acting in $\mathscr{H}=\mathscr{H}_{F}$. The generating elements $b(f), b(g) *$ fulfill:

$$
\begin{aligned}
{\left[b(f), b(g)^{*}\right]_{+} } & =\left(f^{*}, g\right) \mathbb{1}_{F} \\
{[b(f), b(g)]_{+} } & =0
\end{aligned}
$$

where $(\cdot, \cdot)$ is a bilinear form in $\mathscr{K}, \mathbb{1}$ is the unit operator in $\mathscr{U}_{F}(\mathscr{K})$. Let further $\mathscr{U}_{B}(\mathscr{K})$ be a $*$ representation of the CCR algebra over $\mathscr{K}$ acting in some $\mathscr{D} \subset \mathscr{H}=\mathscr{H}_{B}, \mathbb{1}_{B}$ is a unit operator in $\mathscr{U}_{B}(\mathscr{K})$. For generating elements $a(f), a(g)^{*}$ we have

$$
\begin{aligned}
{\left[a(f), a(g)^{*}\right]_{-} } & =\left(f^{*}, g\right) \mathbb{1}_{B} \\
{[a(f), a(g)]_{-} } & =0 .
\end{aligned}
$$


If there exist vacuum vectors $|0\rangle_{F}$ and $|0\rangle_{B}$ respectively:

$$
a(f)|0\rangle_{B}=0=b(f)|0\rangle_{F}
$$

we say about Fock representations.

Let $E_{n}$ be a bounded operator acting on the $n$-th tensor product $\bigotimes_{1}^{n} \mathscr{K}=\mathscr{H}_{n}$ with properties:

$$
E_{n}^{3}=E_{n}, \quad E_{n}^{*}=E_{n}, \quad P_{i k} E_{n}=-E_{n} P_{i k}
$$

where $P_{i k}$ is an operator of permutation of $i$-th and $k$-th $\mathscr{K}$ in $\bigotimes_{1}^{n} \mathscr{K}$. (For a more detailed study of $E_{n}$ as well as for examples, see $[7,8]$.)

Due to $(2.4) E_{n}^{2}$ is a projector and provides the following decomposition of $\mathscr{H}_{n}$ :

$$
\mathscr{H}_{n}=\mathscr{H}_{n} \oplus \mathscr{\mathscr { H }}_{n}
$$

with:

$$
E_{n}^{2} \mathscr{H}_{n}=\mathscr{H}_{n}, \quad\left(1-E_{n}^{2}\right) \mathscr{H}_{n}=\stackrel{2}{\mathscr{H}}_{n} .
$$

Let us decompose additionally $\bigotimes_{1}^{n} \mathscr{K}=\mathscr{H}_{n}$ into the irreducible parts with respect to the symmetry group $\mathscr{S}_{n}$ :

$$
\mathscr{H}_{n}=\sum_{y} Y_{n} \mathscr{H}_{n}
$$

where $Y_{n}$ denotes the Young's operator in $\mathscr{H}_{n}$.

We are able to prove:

Lemma 1. $E_{n}$ is an automorphism of $\mathscr{\mathscr { H }}_{n}$ (a homomorphism $\mathscr{H}_{n} \rightarrow \mathscr{H}_{n}$ with the kernel $\operatorname{ker} E_{n}=\mathscr{\mathscr { H }}_{n}$ ) consisting of isomorphisms

$$
E_{n}: Y_{n} \stackrel{1}{\mathscr{H}}_{n} \leftrightarrow Y_{n}^{*} \stackrel{1}{\mathscr{H}}_{n}
$$

where $Y_{n}^{*}$ is the operator corresponding to the dual Young's scheme.

Proof. Is given in [7], Section 2.

In totally symmetric $\left(Y_{n}=S_{n}\right)$ and antisymmetric $\left(Y_{n}=A_{n}\right)$ cases we have:

$$
E_{n}: \stackrel{1}{\mathscr{H}}_{n}^{F}=A_{n} \stackrel{1}{\mathscr{H}}_{n} \leftrightarrow \stackrel{1}{\mathscr{H}}_{n}^{B}=S_{n} \stackrel{1}{\mathscr{H}}_{n} .
$$

In the present paper we assume (see also [7]):

$$
A_{n} \stackrel{2}{\mathscr{H}}_{n}=0 .
$$

Let $\mathscr{F}$ be given by:

$$
\mathscr{F}=\left\{f=\left\{f_{n}\right\}_{n=0,1, \ldots}, f_{n} \in \mathscr{H}_{n},\|f\|<\infty\right\}=\bigoplus_{1}^{\infty} \mathscr{H}_{n} .
$$

Here $\mathscr{H}_{0}=\mathbb{C}$.

Now:

$$
\mathscr{F}_{B}=\stackrel{1}{\mathscr{F}}_{B} \oplus \stackrel{2}{\mathscr{F}}_{B}, \quad \mathscr{F}_{F}=\stackrel{1}{\mathscr{F}}_{F} \oplus \stackrel{2}{\mathscr{F}}_{F}
$$


with:

$$
\stackrel{i}{\mathscr{F}}_{B}=\bigoplus_{n=0}^{\infty} \stackrel{i}{\mathscr{H}_{n}^{B}}, \quad \stackrel{i}{\mathscr{F}}_{F}=\bigoplus_{n=0}^{\infty} \stackrel{i}{\mathscr{H}^{F}}
$$

where:

$$
\left\|f_{B}\right\|^{2}=\sum_{n=0}^{\infty}\left\|f_{n}^{B}\right\|^{2}
$$

and:

$$
\left\|f_{F}\right\|^{2}=\sum_{n=0}^{\infty}\left\|\hat{f}_{n}^{F}\right\|^{2}=\sum_{n=0}^{\infty}\left\|\hat{f}_{n}^{B}\right\|^{2}=\left\|\hat{f}_{B}\right\|^{2}
$$

if:

$$
f_{n}^{F}=E_{n} f_{n}^{B} .
$$

Here the assumption (2.10) is used.

\section{Results}

All the considerations of the previous section are applicable without any change to more complicated cases $\left(\mathscr{K}=\bigoplus_{1}^{N} k\right)$ admitting internal degrees of freedom in the theory. This fact was proved in [8]. Therefore we restrict ourselves to the scalar case.

Let $E_{n}\left(\boldsymbol{k}_{n}, \boldsymbol{p}_{n}\right)$ be an integral kernel of the $E_{n}$ introduced above, $f_{n m}\left(\boldsymbol{k}_{n}, \boldsymbol{p}_{m}\right)$ be the $n+m$-point symmetric function, $\boldsymbol{k}_{n}=\left(k_{1}, \ldots, k_{n}\right), \boldsymbol{p}_{m}=\left(p_{1}, \ldots, p_{m}\right) ; k, p \in \mathbb{R}^{3}$.

Theorem. Given a Fock representation $\mathscr{U}_{B}(\mathscr{K})$ of the CCR algebra acting in $\mathscr{D} \subset \mathscr{F}_{B},\left(a^{*}, a\right)=\int d k a^{*}(k) a(k)$. Assume the properties (2.4) and (2.10) for $E_{n}$.

Operators $b(f), b(g)^{*}$ with:

$$
\begin{aligned}
b(f)= & : \exp \left\{-\left(a^{*}, a\right)\right\} \sum_{n m} \frac{1}{\sqrt{n ! m !}} \int d \boldsymbol{k}_{n} \int d \boldsymbol{p}_{m} f_{n m}\left(\boldsymbol{k}_{n}, \boldsymbol{p}_{m}\right) \\
& \cdot \stackrel{*}{a}\left(k_{1}\right) \ldots \stackrel{*}{a}\left(k_{n}\right) a\left(p_{1}\right) \ldots a\left(p_{m}\right): \\
= & : \exp \left\{-\left(a^{*}, a\right)\right\} f\left[a^{*}, a\right]:
\end{aligned}
$$

where:

$$
f_{n m}\left(\boldsymbol{k}_{n}, \boldsymbol{p}_{m}\right)=\sqrt{n+1} \delta_{m, 1+n} \int d \boldsymbol{q}_{n} \int d r E_{n}\left(\boldsymbol{k}_{n}, \boldsymbol{q}_{n}\right) f^{*}(r) E_{1+n}\left(r, \boldsymbol{q}_{n}, \boldsymbol{p}_{1+n}\right)
$$

generate a Fock representation $\mathscr{U}_{F}(\mathscr{K})$ of the $C A R$ algebra acting on the following subspace of $\mathscr{F}_{B}: \mathscr{F}_{B}=\bigoplus_{n=0}^{\infty} E_{n}^{2} \mathscr{H}_{n}^{B}$ with the unit operator:

$$
\begin{aligned}
\mathbb{1}_{F}= & : \exp \left\{-\left(a^{*}, a\right)\right\} E^{2}\left[a^{*}, a\right]: \\
= & : \exp \left\{-\left(a^{*}, a\right)\right\} \sum_{n} \frac{1}{n !} \int d \boldsymbol{k}_{n} \int d \boldsymbol{p}_{n} \int d \boldsymbol{r}_{n} \\
& \cdot E_{n}\left(\boldsymbol{k}_{n}, \boldsymbol{r}_{n}\right) E_{n}\left(\boldsymbol{r}_{n}, \boldsymbol{p}_{n}\right) a^{*}\left(k_{1}\right) \ldots a^{*}\left(k_{n}\right) a\left(p_{1}\right) \ldots a\left(p_{n}\right): \\
d \boldsymbol{k}_{n}= & d k_{1} \ldots d k_{n} .
\end{aligned}
$$


Proof. For the proof one can perform an explicit calculation using the Wick rule and taking advantage of the properties of $E_{n}$. We will use a more elegant method, based on the notion of functional representations of the CCR [4-6] and CAR $[8,7]$. The Theorem is formulated in a representation independent way. Therefore it is enough to prove it for any Fock representation. Let us suppose, the notion of functional derivative (Gateaux derivative) with respect to elements of $\mathscr{K}=\mathscr{L}^{2}\left(\mathbb{R}^{3}\right)$ is established, see $[4,5,10]$. For functional power series:

$$
\begin{aligned}
F\left[z^{*}, z\right]= & \sum_{n m} \frac{1}{\sqrt{n ! m !}}\left(f_{n m}, z^{* n} z^{m}\right)=\sum_{n m} \frac{1}{\sqrt{n ! m !}} \int d \boldsymbol{k}_{n} \int d \boldsymbol{p}_{m} \\
& \cdot f_{n m}\left(\boldsymbol{k}_{n}, \boldsymbol{p}_{n}\right) z^{*}\left(k_{1}\right) \ldots z^{*}\left(k_{n}\right) z\left(p_{1}\right) \ldots z\left(p_{m}\right)
\end{aligned}
$$

where $z \in \mathscr{K}$, we assume the following action rule:

$$
\left(F_{1} F_{2}\right)\left[z^{*}, z\right]=F_{1}\left[z^{*}, \frac{d}{d y^{*}}\right] F_{2}\left[y^{*}, z\right]_{\mid y^{*}=0} .
$$

So these double power series can be treated as operators acting in the vector space of single power series $V\left[z^{*}\right]=\sum_{n} \frac{1}{\sqrt{n !}}\left(v_{n}, z^{*}\right)$ due to:

$$
(F V)\left[z^{*}\right]=V^{\prime}\left[z^{*}\right]=F\left[z^{*}, \frac{d}{d y^{*}}\right] V\left[y^{*}\right]_{\mid y^{*}=0} .
$$

How to provide these series a nonformal meaning, see [6]. In this place we mention the following computational rules:

(i) $F_{1}=F_{2}$ if and only if the following holds for all $n, m$

$$
S_{m} S_{n}\left(f_{1}\right)_{m n}\left(\boldsymbol{k}_{m}, \boldsymbol{p}_{n}\right)=S_{m} S_{n}\left(f_{2}\right)_{m n}\left(\boldsymbol{k}_{m}, \boldsymbol{p}_{n}\right)
$$

where $S_{m}$ and $S_{n}$ denote symmetrizations with respect to $\boldsymbol{k}_{m}$ and $\boldsymbol{p}_{n}$ respectively.

(ii) The product $F=F_{1} F_{2}$ is given by

$$
f_{n m}\left(\boldsymbol{k}_{m}, \boldsymbol{p}_{n}\right)=\sum_{l} \int d \boldsymbol{q}_{l}\left(f_{1}\right)_{m l}\left(\boldsymbol{k}_{m}, \boldsymbol{q}_{l}\right) S_{l}\left(f_{2}\right)_{l n}\left(\boldsymbol{q}_{l}, \boldsymbol{p}_{n}\right) \text {. }
$$

We have:

Lemma 2. Let $f_{0} \in \mathbb{C}, \mathscr{L}^{2}\left(\mathbb{R}^{3}\right)=\mathscr{K} \ni f, z$.

The triple $\left\{a, a^{*}, f_{0}\right\}$ with

$$
\begin{aligned}
a(f)\left[z^{*}, z\right] & =\exp \left(z^{*}, z\right) \cdot\left(z, f^{*}\right) \\
a(f)^{*}\left[z^{*}, z\right] & =\exp \left(z^{*}, z\right) \cdot\left(z^{*}, f\right)
\end{aligned}
$$

generates a functional Fock representation of the CCR algebra acting in some $\mathscr{D} \subset \mathscr{F}_{B}$.

Proof. By an explicit use of (3.5), (3.6) we are able to show:

$$
\begin{aligned}
& {\left[a(f), a(g)^{*}\right]_{-}\left[z^{*}, z\right]=\left(f^{*}, g\right) \exp \left(z^{*}, z\right)} \\
& {[a(f), a(g)]_{-}\left[z^{*}, z\right]=0} \\
& a(f) f_{0}=0 .
\end{aligned}
$$

For details see $[4,6]$. 
Lemma 3. Let $f_{0} \in \mathbb{C}, \mathscr{L}^{2}\left(\mathbb{R}^{3}\right)=\mathscr{K} \ni z, f$.

The triple $\left\{b, b^{*}, f_{0}\right\}$ with

$$
b(f)\left[z^{*}, z\right]=\sum_{n, m=0}^{\infty} \frac{1}{\sqrt{n ! m !}}\left(f_{n m}, z^{* n} z^{m}\right),
$$

$f_{n m}\left(\boldsymbol{k}_{n}, \boldsymbol{p}_{m}\right)$ given by the theorem and:

$$
b(f)^{*}\left[z^{*}, z\right]=b(f)\left[z^{*}, z\right]^{*}
$$

generates a functional Fock representation of the CAR algebra in $\stackrel{1}{\mathscr{F}}_{B}$.

Proof. Is given in $[7,8]$, where functional representations of the CAR were first introduced.

$$
\begin{aligned}
{\left[b(f), b(g)^{*}\right]_{+}\left[z^{*}, z\right] } & =\left(f^{*}, g\right) E^{2}\left[z^{*}, z\right] \\
{[b(f), b(g)]_{+}\left[z^{*}, z\right] } & =0 \\
b(f) f_{0} & =0 .
\end{aligned}
$$

In the above Lemmas $\exp \left(z^{*}, z\right)$ and $E^{2}\left[z^{*}, z\right]$ represent unit operators in $\mathscr{U}_{B}(\mathscr{K})$ and $\mathscr{U}_{F}(\mathscr{K})$ respectively.

Lemma 4. Given:

$$
: f\left[a^{*}, a\right]:=\sum_{n, m} \frac{1}{\sqrt{n ! m !}}\left(f_{n m}, a^{* n} a^{m}\right) .
$$

A functional representation of this operator is given by:

$$
: f\left[a^{*}, a\right]:\left[z^{*}, z\right]=\exp \left(z^{*}, z\right) f\left[z^{*}, z\right] .
$$

Proof. By an immediate application of Lemma 2 (see also [6]).

Collecting the Lemmas we are able to prove the theorem. We have by Lemma 4 :

$$
\begin{aligned}
: \exp \left\{-\left(a^{*}, a\right)\right\} f\left[a^{*}, a\right]:\left[z^{*}, z\right] & =\exp \left(z^{*}, z\right) \exp \left\{-\left(z^{*}, z\right)\right\} f\left[z^{*}, z\right] \\
& =f\left[z^{*}, z\right]
\end{aligned}
$$

and:

$$
: \exp \left\{-\left(a^{*}, a\right)\right\} E^{2}\left[a^{*}, a\right]:\left[z^{*}, z\right]=E^{2}\left[z^{*}, z\right]
$$

where the operator $: \exp \left\{-\left(a^{*}, a\right)\right\} E^{2}\left[a^{*}, a\right]$ : is the projection operator onto the subspace $\mathscr{F}_{B}$.

But:

$$
f\left[z^{*}, z\right]=b(f)\left[z^{*}, z\right]
$$

and therefore Lemma 3 implies the canonical anticommutation relations for $b(f)$. The Fock vacuum of $\mathscr{F}_{B}$ is obviously annihilated by $b(f)$. In the above, the domain was $\mathscr{D} \cap \mathscr{F}_{B}^{1}$. Since $b(f)$ is bounded (a general property of the CAR), its closure is defined on the whole space $\mathscr{\mathscr { F }}_{B}$. This complets the proof of the Theorem. 


\section{References}

1. Heisenberg, W.: Introduction to the unified field theory of elementary particles. London: Interscience 1966

2. Streater, R.F., Wilde, I. F.: Nucl. Phys. B 24, 561 (1970)

3. Kalnay, A.J., Mac Cotrina, E., Kademova, K. V.: Int. J. Theor. Phys. 7, 9 (1973)

4. Rzewuski, J.: Field theory, part II. PWN Warsaw: Illife Books Ltd. 1969

5. Berezin,F.A.: Methods of second quantization. Moscow: Nauka 1965 (in Russian)

6. Rzewuski, J.: Rep. Math. Phys. 1, 195 (1971)

7. Garbaczewski,P., Rzewuski, J.: Rep. Math. Phys. 6, 423 (1974)

8. Garbaczewski,P.: Rep. Math. Phys. 7, 9 (1975)

9. Emch,G.G.: Algebraic methods in statistical mechanics and quantum field theory. London: Wiley, Interscience 1972

10. Rohrlich, F.: In: Analytic methods in mathematical physics. Newton, R. G., Gilbert, R.P. (Ed.). New York: Gordon and Breach 1970

11. Jost, R.: The general theory of quantized fields. Moscow: Mir 1967 (in Russian)

Communicated by H. Araki

\author{
Piotr Garbaczewski \\ Institute of Theoretical Physics \\ University of Wrockaw \\ Cybulskiego 36 \\ 50-205 Wrocław, Poland
}

